\title{
A Equoterapia no equilíbrio postural de pessoas com Esclerose Múltipla
}

\author{
Karla Mendonça Menezes¹, Fabiana Moraes Flores², Fernanda de Moraes Vargas², \\ Claudia Morais Trevisan ${ }^{3}$, Fernando Copetti ${ }^{4}$
}

\begin{abstract}
RESUMO
A Esclerose Múltipla (EM) é uma doença crônica e progressiva decorrente de respostas inflamatórias auto-imunes que acometem à mielina do tecido nervoso, sendo comuns na EM as alterações no equilibrio postural. Objetivo: verificar se a Equoterapia, inserida como atividade complementar, influencia o equilíbrio postural de pessoas com EM. Método: Foram avaliados seis casos. O desempenho do equilibrio postural foi avaliado pela Escala de Equilíbrio de Berg (EEB) antes e após 30 sessões de Equoterapia, realizadas duas vezes por semana, em um período de quatro meses. Resultados: Após a intervenção observou-se aumento dos escores na EEB em quatro casos e manutenção em dois. Conclusões: A estimulação da Equoterapia, como atividade terapêutica complementar, mostrou-se capaz de melhorar ou manter os resultados no desempenho das tarefas funcionais que envolvem o equilíbrio estático e dinâmico de pessoas com EM avaliadas pela EEB.
\end{abstract}

Descritores: Terapia Assistida por Cavalos; Esclerose Múltipla; Equilíbrio Postural.

\section{Hippotherapy in postural balance of Multiple Sclerosis patients}

\begin{abstract}
Multiple sclerosis (MS) is a chronic and progressive disease induced by autoimmune inflammatory responses that attack myelin of the nerve tissue, with changes in postural balance being common manifestations in MS. Objective: Verify if the hippotherapy, inserted as a complementary activity, influences the postural balance of MS patients. Method: A total of six cases were evaluated. The performance of postural balance was assessed by the Berg Balance Scale (BBS) before and after 30 sessions of hippotherapy, performed twice a week, over a period of four months. Results: After the intervention, there was an increase in scores on BBS in four individuals and two of them maintained their scores. Conclusions: The stimulation of hippotherapy as a complementary activity proved to be able to improve the results of performance in functional tasks that require static and dynamic balance, assessed by BBS.
\end{abstract}

Descriptors: Equine-Assisted Therapy; Multiple Sclerosis; Postural Balance.

\footnotetext{
${ }^{1}$ Mestre em Ciências da Saúde pela Universidade Federal de Santa Maria (UFSM), Santa Maria, RS, Brasil.

${ }^{2}$ Especialista em Atividade Física, Desempenho Motor e Saúde pela Universidade Federal de Santa Maria (UFSM), Santa Maria, RS, Brasil.

${ }^{3}$ Doutora em Ciências da Saúde pela Universidade Federal de Santa Maria (UFSM), Santa Maria, RS, Brasil.

${ }^{4}$ Doutor em Ciência do Movimento Humano pela Universidade Federal de Santa Maria (UFSM), Santa Maria, RS, Brasil.
} 


\section{Introdução}

A Esclerose Múltipla (EM) é uma doença neurológica crônica caracterizada pela infiltração de células inflamatórias no Sistema Nervoso Central (SNC) causando lesões à mielina que podem interromper a transmissão de impulsos nervosos ou acarretar falha na propagação de potenciais de ação axonais ${ }^{1}$. A EM geralmente tem caráter heterogêneo e tende a evoluir através de episódios de surto-remissão resultando em formas clínicas distintas, conforme a localização e sequência temporal das lesões ${ }^{1}$. As manifestações da EM variam de um indivíduo para outro, mas, invariavelmente, afetam a capacidade funcional através de diversas deficiências, incluindo fraqueza, desequilíbrio, fadiga, tremor e espasticidade².

Estudos epidemiológicos apontam a EM como uma das principais causas de déficit neurológico em adultos jovens, causando problemas físicos, intelectuais e emocionais. Esses estudos revelaram taxas de 15 casos/100.000 habitantes nas regiões sul e sudeste do Brasili,4. Em especial, um recente estudo ${ }^{5}$ apontou a prevalência de EM em 27,2 casos/100.000 na cidade de Santa Maria - RS, Brasil, demonstrando um índice de incidência desta doença muito superior a média nacional.

Alguns estudos sugerem que a utilização de intervenções terapêuticas podem favorecer a funcionalidade e tem potencial para melhorar muitas das deficiências observadas na $E M^{6-8}$. No entanto, devido à multiplicidade de funções comprometidas e o curso imprevisível e progressivo da EM, a efetividade dessas intervenções nem sempre é comprovada em aspectos funcionais ${ }^{9}$. A avaliação de aspectos relativos à capacidade funcional, entre eles o equilíbrio corporal, permitem selecionar a prática terapêutica adequada e mensurar os resultados da intervenção proposta ${ }^{6}$.

Uma variedade de técnicas laboratoriais e escalas clínicas são utilizadas na avaliação do equilíbrio corporal. Dentre elas, as escalas ganharam popularidade na comunidade clínica e científica por permitir a comparações entre grupos de sujeitos com diversas patologias, facilidade de aplicação clínica e valores mais acessíveis quando comparados às avaliações laboratoriais. A Escala de Equilíbrio de Berg $(E E B)^{10}$ é uma importante ferramenta para avaliação do equilíbrio funcional em pessoas com EM apresentando nível de confiabilidade quando comparado a outros métodos ${ }^{11}$, sendo adaptada culturalmente para o Brasil em 2004'12.

Abordagens terapêuticas como a fisioterapia convencional6, reabilitação vestibular ${ }^{7}$ e 0 treinamento de equilíbrio ${ }^{8}$ vem apresentando resultados positivos na tentativa de atenuar os comprometimentos desencadeados pela EM. Esses estudos apontam que o treinamento do equilíbrio deve ser componente do programa de reabilitação em pessoas com $\mathrm{EM}^{6,8}$, sendo que exercícios que utilizam estratégias sensoriais apresentaram melhores respostas no equilíbrio funcional do que exercícios aeróbicos e resistidos ${ }^{13,14}$.

A equitação com fins terapêuticos, ou terapia assistida por cavalos, no Brasil denominada Equoterapia, tem sido utilizada como complementar para várias condições que comprometem o equilíbrio postural. Em pessoas com EM os efeitos da Equoterapia foram descritos em alguns estudos que avaliaram equilíbrio estático ${ }^{15}$ e dinâmico ${ }^{16-19}$, marcha ${ }^{16,17}$, coordenaçãa ${ }^{17}$, atividades da vida diária ${ }^{17}$ e qualidade de vida ${ }^{17,20}$ e evidenciaram efeitos positivos nas variáveis investigadas. Dois desses estudos ${ }^{17,18}$ utilizaram a EEB para avaliar o equilíbrio e apontaram efeitos positivos após a intervenção com Equoterapia. No entanto, ambos salientaram que, devido à diversidade de sintomas e comprometimentos desencadeados pela EM, há necessidade de mais estudos que ajudem na compreensão sobre a eficácia dessa estimulação.

Considerando a alta prevalência de casos de EM na cidade de Santa Maria ${ }^{5}$ e o potencial terapêutico da Equoterapia, descrito em várias condições que comprometem o equilíbrio postural, esse estudo se propôs a verificar se a Equoterapia, inserida como atividade terapêutica complementar, é capaz de alterar o equilíbrio postural de pessoas com EM.

\section{Metodologia}

\section{Delineamento do Estudo}

Esse estudo transversal teve delineamento experimental, sem grupo controle, do tipo estudo de casos.

\section{$\underline{\text { Sujeitos }}$}

Os sujeitos desse estudo foram selecionados por conveniência, de forma não probabilística. $O$ grupo de abrangência deste estudo foi os participantes da Associação dos Portadores de Esclerose Múltipla de Santa Maria e Região (APEMSMAR). Todos os integrantes da APEMSMAR foram esclarecidos quanto aos objetivos e método do estudo. Os critérios de inclusão foram: apresentar diagnóstico neurológico de EM acompanhado de queixa de instabilidade postural. 
Estar na faixa etária entre 30 e 60 anos e possuir autonomia para andar, com ou sem dispositivos de auxílio. Além disso, apresentar liberação médica para a prática de Equoterapia e não ter contato prévio com atividades que envolvessem estimulações equestres. $O$ critério de exclusão foi ter frequência inferior a $70 \%$ das sessões previstas.

Foi conduzida uma anamnese para a triagem dos sujeitos, bem como identificação das características individuais e histórico clínico. Inicialmente quatorze sujeitos preencheram aos critérios previamente expostos. No entanto, somente sete manifestaram interesse em participar do estudo. Estes foram avaliados pela EEB e inseridos nas atividades da Equoterapia. Foi excluído da análise um sujeito que não atingiu a frequência mínima das sessões previstas. Dessa forma, seis casos foram analisados individualmente, utilizando o sujeito como seu próprio controle.

\section{Procedimentos éticos}

Os métodos de avaliação e protocolos de intervenção utilizados foram aprovados pelo Comitê de Ética em Pesquisa da Universidade Federal de Santa Maria e acompanham as normas da Resolução 196/96 do Conselho Nacional de Saúde sobre pesquisa envolvendo seres humanos, conforme parecer 0146.0.243.000-09.

\section{Avaliação do equilíbrio postural}

A avaliação do equilíbrio postural foi realizada através da Escala de Equilíbrio de Berg (EEB) antes e após o período de intervenção. A EEB é composta por 14 tarefas comuns da vida diária que envolvem o equilíbrio estático e dinâmico. Essa escala permite monitorar o estado do equilíbrio, o curso de uma doença, predizer quedas, selecionar pacientes aptos ao processo de reabilitação e a resposta do ao tratamento ${ }^{21}$.

As tarefas da EEB são avaliadas através de observação e possuem uma escala ordinal de cinco alternativas, variando de zero a quatro, totalizando um escore máximo de 56 pontos. Estes pontos são subtraídos caso o tempo ou a distância não sejam atingidos ou se o sujeito necessitar auxílio para execução da tarefa. 0 desempenho é classificado em 0 (incapaz de executar) a 4 pontos (normal) em 14 situações em atividades de vida diária, tais como, ficar de pé, levantarse, andar, inclinar-se à frente, transferir-se, virar-se, dentre outras, de acordo com o grau de dificuldade. 0 declínio nas pontuações dessa escala está associado a um risco elevado de quedas ${ }^{12,21-24}$.

\section{Equoterapia}

Os sujeitos foram submetidos a duas sessões semanais de Equoterapia durante um período de quatro meses, totalizando 30 sessões, com duração média de 50 minutos incluindo o tempo de aproximação, montaria e apeio, sendo cumpridos 30 minutos contínuos de atividades com o cavalo em movimento andando ao passo. As sessões foram estruturadas com objetivo de estimular o equilíbrio dos sujeitos. Desse modo, com o sujeito montado e com o cavalo em movimento, foram utilizadas variações na velocidade da andadura do cavalo, no tipo de piso, mudanças de direção e de combinações de movimentos. Além disso, durante a estimulação foram realizados exercícios com balizas para realizar movimentos e zigue-zague, troca de cadência da andadura do cavalo, bem como atividades que envolviam 0 deslocamento do cavalo realizando figuras de picadeiro. Para estas atividades, o nível de dificuldade das tarefas foi aumentado gradativamente, respeitando a capacidade individual de cada participante quanto ao seu limite de execução.

Os sujeitos deste estudo realizavam tratamento medicamentoso e de fisioterapia convencional há pelo menos seis meses. Dessa forma, cabe salientar que as atividades de estimulação da Equoterapia foram incluídas no cotidiano dos sujeitos como um método terapêutico complementar. As atividades de fisioterapia, na qual os sujeitos relatavam participar, foram controladas quanto ao número de vezes realizadas durante o período do estudo. As atividades desenvolvidas durante as sessões de fisioterapia convencional não foram controladas.

As sessões de intervenção foram conduzidas por profissionais e acadêmicos da área da saúde capacitados para trabalho com Equoterapia. As atividades foram realizadas em duplas, sendo priorizada a manutenção do mesmo cavalo para cada praticante ao longo do tratamento. Dessa forma, foram utilizados dois cavalos treinados para 0 trabalho em Equoterapia: uma fêmea, cinco anos, sem raça definida, $155 \mathrm{~cm}$ à altura da cernelha, massa corporal de $480 \mathrm{~kg}$ e um macho, 10 anos, raça quarto de milha, $158 \mathrm{~cm}$ à altura da cernelha, $510 \mathrm{~kg}$ de massa corporal, ambos com característica de passada de sobrepistar. Os cavalos estiveram equipados com manta, estribos abertos e rédeas para montaria e foram conduzidos por auxiliares guias. Todos os procedimentos de segurança adotados durante as sessões seguiram as orientações da Associação Nacional de Equoterapia (ANDE-Brasil), no que se refere ao uso de capacete e vestimenta adequada. 


\section{Resultados}

A anamnese revelou que todos os sujeitos recebiam terapia imunossupressora corrente e participavam de sessões semanais de fisioterapia convencional há pelo menos seis meses. O número de surtos que ocorreram desde o diagnóstico não estava disponível. Os dados clínicos e demográficos dos sujeitos desse estudo são apresentados na Tabela 1.

Tabela 1 - Caracterização dos Sujeitos

\begin{tabular}{c|c|c|c|c|c}
\hline ID & Sexo & $\begin{array}{c}\text { Idade } \\
\text { (anos) }\end{array}$ & $\begin{array}{c}\text { MC } \\
(\mathbf{K g})\end{array}$ & $\begin{array}{c}\text { Estatura } \\
(\mathbf{m})\end{array}$ & $\begin{array}{c}\text { TD } \\
\text { (anos) }\end{array}$ \\
\hline I & $\mathrm{F}$ & 51 & 75,1 & 1,70 & 3 \\
\hline $\mathrm{II}$ & $\mathrm{F}$ & 35 & 49,5 & 1,65 & 14 \\
\hline $\mathrm{III}$ & $\mathrm{F}$ & 32 & 67,8 & 1,69 & 4 \\
\hline $\mathrm{IV}$ & $\mathrm{F}$ & 58 & 57,6 & 1,43 & 28 \\
\hline $\mathrm{V}$ & $\mathrm{M}$ & 40 & 101 & 1,70 & 6 \\
\hline $\mathrm{VI}$ & $\mathrm{F}$ & 47 & 64 & 1,51 & 1 \\
\hline
\end{tabular}

ID - identificação; MC - Massa Corporal; TD - Tempo de Diagnóstico de EM;

Nota: De acordo com diagnóstico neurológico, os sujeitos não possuíam classificação definida quanto ao tipo de EM.

O equilíbrio funcional dos sujeitos com EM foi avaliado pela EEB antes e após quatro meses de intervenção com Equoterapia. $O$ desempenho dos sujeitos foi analisado individualmente e pode ser visualizado na Figura 1.

Os resultados apresentados na Figura 1 demonstram que quatro dos seis casos investigados aumentaram os escores na EEB após a intervenção com a Equoterapia. O sujeito I teve uma variação de 10 pontos no escore da EEB após a intervenção. $\mathrm{O}$ sujeito II aumentou em três pontos o escore inicial da EEB e os sujeitos IV e $\mathrm{V}$ aumentam dois e cinco pontos, respectivamente. Os sujeitos III e VI, que apresentaram os melhores desempenhos na avaliação inicial, mantiveram o mesmo escore na EEB após o período com a estimulação da Equoterapia.

Figura 1 - Desempenho individual dos sujeitos na EEB antes e após intervenção com Equoterapia

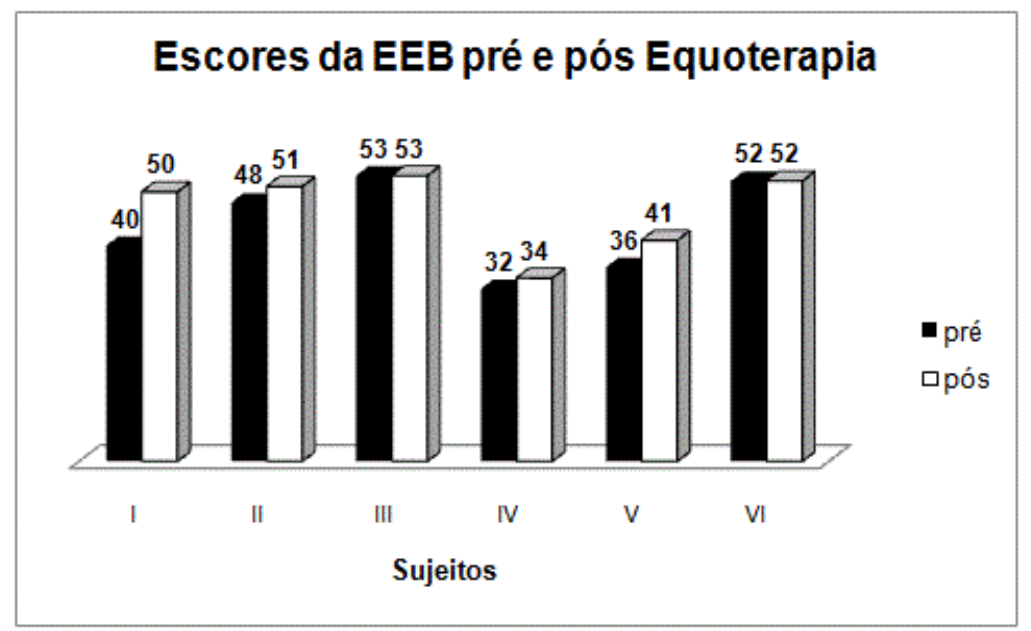

\section{Discussão}

Este estudo se propôs a avaliar o equilíbrio funcional de pessoas com EM após intervenção com Equoterapia. Aanálise descritiva dos resultados indica que quatro dos seis sujeitos avaliados melhoraram os escores do equilíbrio funcional, medido através da EEB, enquanto os dois outros sujeitos mantiveram os escores após a intervenção. 
A EEB é uma avaliação funcional do desempenho do equilíbrio, baseada em 14 itens comuns do dia a dia. A pontuação máxima a ser alcançada é de 56 pontos ${ }^{10,12,21-24}$. No estudo original de Berg et al. ${ }^{10}$, o ponto de corte em 45 é indicado para calcular as estimativas de risco de quedas. Nesse estudo, três sujeitos (l, VI, V) apresentam escores iniciais inferiores a 45 pontos na testagem inicial. Após quatro meses, com duas sessões semanais de Equoterapia, esses sujeitos aumentaram o escore inicial, sendo que o sujeito I foi capaz de aumentar sua pontuação e sair da zona de risco de quedas. Além disso, observamos que três sujeitos (II, III e VI) apresentaram escore superior a 45 pontos na testagem inicial. Dentre eles, os sujeitos II e VI não alteraram o desempenho na EEB após a intervenção tendo apresentado escore elevado no pré-teste, 53 e 52 pontos, respectivamente evidenciando um menor grau de comprometimento do equilíbrio postural e indicando menor risco de queda.

Por se tratar de uma disfunção progressiva, os comprometimentos associados à EM tendem a aumentar gradativamente. Indicativos de deterioração funcional, mesmo na ausência de recidiva clínica, foram observados em indivíduos recémdiagnosticados com $\mathrm{EM}^{25}$. Na tentativa de minimizar esses comprometimentos e manter a capacidade funcional, são utilizadas diferentes estratégias de reabilitação como a fisioterapia convencional, treinamento de equilíbrio, reabilitação vestibular, exercícios aeróbicos e exercícios resistidos, por exemplo.

Estudos que investigam o efeito da fisioterapia convencional ${ }^{6}$, reabilitação vestibular ${ }^{7}$ e treinamento de equilíbrio ${ }^{8}$ observaram melhoras nos níveis de independência funcional e equilíbrio. Cattaneo et al. ${ }^{13}$ ao comparar a efetividade de diferentes estímulos terapêuticos, observaram que exercícios que utilizam estratégias sensoriais desencadearam melhores respostas no equilíbrio funcional do que exercícios aeróbicos e resistidos.

Resultados positivos de intervenções através da Equoterapia foram descritas em distintas condições neurológicas que comprometem o controle postural e a mobilidade ${ }^{15-19,26-28}$. Especificamente na $\mathrm{EM}$, dois estudos, que utilizaram a EEB para avaliar o equilíbrio funcional, após intervenção com Equoterapia foram encontrados ${ }^{17,18}$. Mesmo com dosagens diferentes de tratamento, ambos os estudos apontaram melhora no equilíbrio após o período de intervenção.

Em nosso estudo, quatro dos seis sujeitos avaliados aumentaram a pontuação na EEB, indicando melhora no desempenho funcional do equilíbrio, após quatro meses de intervenção com a Equoterapia. Os outros dois sujeitos não modificaram os escores após a intervenção. Esses sujeitos apresentaram valores próximos à pontuação máxima na $E E B$, indicando bom desempenho nas tarefas, já no teste inicial. Os resultados encontrados nesse estudo convergem aos achados dos estudos anteriores que apontam a Equoterapia como uma importante forma de estimulação do equilíbrio postural de pessoas com EM.

Algumas limitações importantes no desenvolvimento desse estudo precisam ser consideradas. Essas residem principalmente na dificuldade de homogeneizar grupos de pessoas com disfunções neurológicas e na ausência de definição no diagnóstico clínico quanto ao tipo de EM. Além disso, a ausência de um grupo controle e o monitoramento das atividades realizadas nos outros de tratamentos também devem ser considerados. Nesse sentido, mesmo considerando que os sujeitos tinham níveis de comprometimentos distintos entre eles, a intervenção parece ter influenciado positivamente 0 equilíbrio funcional dos sujeitos investigados. Observou-se ainda que quando o comprometimento no equilíbrio mostrouse pouco evidente a intervenção não foi capaz de melhorar o desempenho quando avaliado pela EEB. No entanto, vale ressaltar que, se tratando de uma desordem progressiva, a manutenção dos escores que estavam em bom nível, num período de quatros meses, é um fator que merece ser considerado.

Arealização de estudos randomizados e controlados são sempre recomendados para aumentar o rigor e a confiabilidade dos resultados da intervenção proposta. No entanto, mesmo não sendo possível realizar esse delineamento para este estudo, cabe destacar que a utilização do sujeito como seu próprio controle, antes e após a Equoterapia foi útil para a avaliação dos efeitos no equilíbrio postural. No entanto, considerar a incidência de quedas e ou os riscos de queda, em vez de somente a queixa de instabilidade postural, pode ser um delineamento que traga resultados clinicamente significativos para os estudos futuros com esta população.

\section{Considerações Finais}

Considerando que a EM é uma doença progressiva e que os tratamentos clínicos e de reabilitação buscam interferir em sua evolução, a inserção de recursos terapêuticos que busquem minimizar os efeitos negativos da doença são importantes aliados. Neste sentido, mesmo considerando que os sujeitos tinham níveis de comprometimentos distintos, a prática da Equoterapia, oportunizada como uma atividade complementar, parece ter influenciado positivamente o equilíbrio funcional dos sujeitos. 
Os achados deste estudo reforçam a literatura que ampara o uso da Equoterapia como uma intervenção com potencial para tratamento do equilíbrio postural de pessoas com EM.

\section{Referências Bibliográficas}

1. Korneck B, Lassmann H. Neuropathology of multiple sclerosis - new concepts. Brain Res Bull 2003; 61(3):321-6.

2. Frankel D. Esclerose Múltipla. In: Umphered DA. Reabilitação neurológica. $4^{a}$ ed. Barueri, SP: Manole, 2004.

3. Finkelsztejn A, Cristovam R, Moraes G, Lopes M, Silva A, Garcia M, et al. Clinical Features of Multiple Sclerosis in the South of Brazil. Arq Neuropsiquiatr. 2009; 67(4):1071-5.

4. Papais-Alvarenga R, Santos C, Abreu J, Siqueira H, Camargo S, Almeida A, et al. Esclerose Múltipla (EM): Perfil clínico e evolutivo no município do Rio de Janeiro. Rev bras neurol. 1995; 31(2):75-87.

5. Finkelsztejn A, Lopes JS, Noal J, Finkelsztejn JM. The prevalence of multiple sclerosis in Santa Maria, Rio Grande do Sul, Brazil. Arq Neuropsiquiatr. 2014; 72(2):104-6.

6. Rodrigues, IF, Nielson MBP, Marinho AR. Avaliação da fisioterapia sobre o equilíbrio e a qualidade de vida em pacientes com esclerose múltipla. Rev Neurocienc. 2008; 16(4): 269-74.

7. Pavan K, Marangoni BEM, Schmidt KB, Cobe FA, Matuti GS, Nishino LK et al. Reabilitação vestibular em pacientes com Esclerose Múltipla remitente- recorrente. Arq Neuropsiquiatr 2007; 65(2-A):332-5.

8. Almeida SEM, Bensuaski K, Cacho EWA, Oberg TD. Eficiência do treino de equilíbrio na Esclerose Múltipla. Fisioterapia em Movimento. 2007; 20(2):41-8.

9. Giesser B. Exercise Training in Persons with Multiple Sclerosis. US Neurological Disease. 2007:37-9.

10. Berg KO, Norman KE. Functional assessment of balance and gait. Clin Geriatr Med 1996; 12(4):705-23.

11. Cattaneo D, Jonsdottir J, Repetti S. Reliability of four scales on balance disorders in persons with multiple sclerosis. Disabil Rehabil. 2007; 29(24):1920-25.

12. Miyamoto ST, Junior L, Berg KO et al. Brazilian version of the Berg balance scale. Braz J Med Biol Res 2004; 37(9):1411-21.

13. Cattaneo D, Jonsdottir J. Sensory impairments in quiet standing in subjects with multiple sclerosis. Mult Scler. 2009; 15(1):59-67.

14. Cattaneo D, Jonsdottir J, Zocchi M, Regola A. Effects of balance exercises on people with multiple sclerosis: a pilot study. Clin Rehabil 2007; 21:771-81.

15. Menezes KM, Copetti F, Wiest MJ, Trevisan, CM, Silveira, AF. Effect of hippotherapy on the postural stability of patients with multiple sclerosis: a preliminary study. Fisioter Pesq. 2013;20(1):43-49

16. Muñoz-Lasa S FG, Valero R, Gomez-Muñiz F, Rabini A, Varela E. . Effect of therapeutic horseback riding on balance and gait of people with multiple sclerosis. G Ital Med Lav Erg 2011;33(4): 462-7

17. Hammer A, Nilsagard Y, Forsberg A, Pepa H, Skargren E, Oberg B. Evaluation of therapeutic riding (Sweden)/ hippotherapy (United States). A single-subject experimental design study replicated in eleven patients with multiple sclerosis. Physiother Theory and Pract. 2005; 21(1):51-77.

18. Silkwood-Sherer $\mathrm{D}$, Warmbier $\mathrm{H}$. Effects of hippotherapy on postural stability, in persons with multiple sclerosis: a pilot study. JNPT. 2007; 31(2):77-84.

19. Frevel D, Mäurer M. Internet-based home training is capable to improve balance in multiple sclerosis: a comparative trial with hippotherapy. Eur J Phys Rehabil Med. 2014:1-9.

20. Flores FM, Sousa LS, Menezes KM, Copetti F, Trevisan CM. Quality of life in multiple sclerosis patients participating in therapeutic horseback riding. ConScientiae Saúde, 2014; 13(1):39-46.

21. Halssa KE, Brovold T, Graver V, Sandvik L, Bergland A. Assessments of interrater reliability and internal consistency of the Norwegian version of the Berg Balance Scale. Arch Phys Med Rehabil 2007; 88(1):94-8.

22. Ribeiro AS, Pereira JS. Balance improvement and reduction of likelihood of falls in older women after Cawthorne and Cooksey exercises. Braz J Otorhinolaryngol 2005; 71(1):38-46.

23. Azevedo ERFBM, Macedo LS, Paraízo MFN, Oberg TD, Lima NMFV, Cacho EWA. Correlação do déficit de equilíbrio, comprometimento motor e independência funcional em indivíduos hemiparéticos crônicos. Acta Fisiatr. 2008; 15(4): 225-8.

24. Mao HF, Hsueh IP, Tang PF, Sheu CF, Hsieh CL. Analysisand comparison of the psychometric properties of three balance measures for stroke patients. Stroke 2002; 33(4):1022-27.

25. Martin CL, Galea MP, Phillips BA, Kilpatrick TJ, Butzkueven H. Gait and balance parameters as clinical markers of 
disease progression in early multiple sclerosis. In: 18th Meeting of the European Neurological Society; 2008.

26. Benda W, McGibbon NH, Grant KL. Improvements in muscle symmetry in children with cerebral palsy after equineassisted therapy (hippotherapy). J altern complement med 2003; 9(6):817-25.

27. McGibbon NH, Benda W, Duncan BR, Silkwood-Sherer D. Immediate and Long-Term Effects of Hippotherapy on symmetry of adductor muscle activity and functional ability in children with spastic cerebral palsy. Arch Phys Med Rehabil 2009; 90(6):966-74.

28. Beinotti F, Correia N, Christofoletti G, Borges G. Use of hippotherapy in gait training for hemiparetic post-stroke.Arq Neuropsiquiatr. 2010; 68(6):908-13.

\section{Karla Mendonça Menezes}

Endereço para correspondência - Rua: Radialista Oswaldo Nobre, n 660, bloco 04, apartamento 315,

Bairro: Prado, CEP 97035 000, Cidade: Santa Maria, RS, Brasil.

E-mail: karlam_ef@yahoo.com.br

Lattes: http://lattes.cnpq.br/9887976587039884

Fabiana Moraes Flores - fabiana_moraes@yahoo.com.br

Fernanda de Moraes Vargas - fernandadevargas@hotmail.com

Claudia Morais Trevisan - claudiamoraistrevisan@gmail.com

Fernando Copetti - copettif@gmail.com

\section{Enviado em 21 de junho de 2014.} Aceito em 15 de dezembro de 2014. 
